\title{
Private Information: Similarity as Compatibility
}

\author{
João Correia-da-Silva ${ }^{1}$ \\ FCT. Faculdade de Economia. Universidade do Porto. PORTUGAL \\ Carlos Hervés-Beloso ${ }^{2}$ \\ RGEA. Facultad de Económicas. Universidad de Vigo. SPAIN.
}

September 16th, 2004

\begin{abstract}
We investigate the continuity of equilibrium in differential information economies with a finite number of agents. In this setting, agents can make contingent contracts based on events that are commonly observed. With private information modeled as finite partitions of a compact and metrizable space of states of nature, we introduce a topology on information that evaluates the similarity between private information fields taking into account their compatibility. This topology allows us to establish upper semicontinuity of the private core correspondence.
\end{abstract}

Keywords: Differential Information Economy, Asymmetric Information, Radner Equilibrium, Private Core, Topologies on Information.

JEL Classification Numbers: C62, D50, D82, G13.

\footnotetext{
${ }^{1}$ João Correia-da-Silva (e-mail: joaocs@sapo.pt) acknowledges support by Research Grant SFRH/BD/11435/2002 from Fundação para a Ciência e Tecnologia, Ministério da Ciência e Tecnologia.

${ }^{2}$ Carlos Hervés-Beloso (e-mail: cherves@uvigo.es) acknowledges support by Research Grant BEC2003-09067-C04-01 (Ministerio de Ciencia y Tecnología and FEDER)
} 


\section{Introduction}

A classic problem in economic theory is that of the continuity of economic behavior with respect to variations in agents' characteristics. Economies with similar agents are expected to generate similar outcomes. In the Arrow-Debreu setting, where agents are characterized by preferences and initial endowments, Kannai (1970) and Hildenbrand and Mertens (1972) have, respectively, shown upper semicontinuity of the core and Walrasian equilibrium correspondences. In differential information economies, agents are also characterized by their private information, so similarity between agents also requires proximity of private information, evaluated by some topology on the information fields.

In this paper, as in the previous literature, information is modeled as a finite partition on the states of nature such that an agent distinguishes states of nature that belong to different sets of the partition. The question we seek to answer is: How does an economy respond to small changes in the characteristics of the agents, including information? To pursue this inquiry, we need a precise notion of proximity between information fields. Boylan (1971) proposed a topology that is analogous to the Hausdorff metric on closed sets. Allen (1983) studied its properties and proved convergence of consumer demand and continuity of indirect utility with respect to this topology on information. Cotter $(1986,1987)$ introduced a weaker topology, based on the pointwise convergence metric, and showed that it retains the same properties of continuity of conditional expected utility and excess demand.

In differential information economies, the continuity problem is not vacuous. Under general conditions, the Radner equilibrium set and the private core are not empty. Existence of competitive equilibrium in differential information economies was established by Radner $(1968,1982)$, while the first cooperative concepts were introduced by Wilson (1978): the "fine core" and the "coarse core". In the 
"fine core", agents share all information inside the blocking coalition, while in the "coarse core", the blocking coalition may only use the common information. Considering that agents may not have the possibility or interest in sharing information, Yannelis (1991) introduced the notion of "private core" and established its non-emptiness in general conditions. Our preference for this concept is further justified by a result of Einy, Moreno and Shitovitz (2001) that establishes equivalence between private core and Radner equilibrium in economies with a continuum of agents.

In this general equilibrium framework, Einy, Haimanko, Moreno and Shitovitz (2004) used the metric of Boylan to establish upper semicontinuity of the Radner equilibrium correspondence. They also presented an example showing failures of upper semicontinuity of the private core, which is also valid with the weaker topology of Cotter. This failure of upper semicontinuity is an unpleasant result that brings doubts on the adequacy of the topologies of Boylan and Cotter on information fields.

We investigate the continuity properties of the private core and Walrasian equilibrium correspondences in differential information economies with a finite number of agents, where the private information of each agent is a finite partition of a compact and metrizable space of states of the world. This is the setting of the negative example of Einy et al (2004) that excludes upper semicontinuity of the private core correspondence. Our contribution is the introduction of a topology on information in which neighboring information fields are compatible, in the sense that they allow essentially the same contingent contracts. This topology allows us to establish the upper semicontinuity of the private core. We also show that with private information restricted to finite partitions, the continuity of Radner equilibrium result of Einy et al (2004) is still true when the uniform continuity of the utility functions is relaxed.

The rest of the paper is organized as follows: in section 2 the differential informa- 
tion economy is defined, in section 3 the topology on information is introduced, section 4 establishes upper semicontinuity of the private core and Radner equilibrium correspondences, and section 5 concludes the paper with an illustration and some remarks.

\section{The Differential Information Economy}

Our framework is the model of a pure exchange economy with differential information (see Radner $(1968,1982)$ ). We consider a finite number of agents and a finite number of goods, $\ell$, in each state. $N$ is the set of $n$ traders or agents and, in every state of nature, the commodity space is the positive orthant of $\mathbb{R}^{\ell}$.

The economy extends over two time periods. Consumption takes place in the second period. In the first, there is uncertainty over the states of nature and agents make contracts that may be contingent on state of nature that occurs in the second period (ex-ante contract arrangement).

Let $(\Omega, \mathcal{B}, \mu)$ be a probability measure space, describing the exogenous uncertainty, where:

- $\Omega$, compact and metrizable, denotes the possible states of nature;

- $\mathcal{B}$, a $\sigma$-algebra of subsets of $\Omega$, denotes the set of all events;

- $\mu$, a countably additive probability measure on $(\Omega, \mathcal{B})$, gives the (common) prior of every agent.

In our model of a differential information exchange economy with a finite number of agents, $\mathcal{E} \equiv\left(e^{i}, u^{i}, \mathcal{F}^{i}\right)_{i=1}^{n}$, for each agent $i$ it is assumed that: 
1. A finite partition of $\Omega, P_{i}$ generates the $\sigma$-algebra $\mathcal{F}^{i} \subset \mathcal{B}$, the private information of agent $i$.

2. $u^{i}: \Omega \times \mathbb{R}_{+}^{\ell} \rightarrow \mathbb{R}_{+}$is the random utility function of agent $i$. For all $\omega$, $u^{i}(\omega, \cdot): \mathbb{R}_{+}^{\ell} \rightarrow \mathbb{R}_{+}$is continuous, strictly monotone and concave. And for every $x, u^{i}(\cdot, x): \Omega \rightarrow \mathbb{R}_{+}$is measurable with respect to $\mathcal{B}$, bounded, and continuous in the interior of elements of $P_{i}$.

3. $e^{i}: \Omega \rightarrow \mathbb{R}_{+}^{\ell}$, representing the random initial endowments of agent $i$, is $\mathcal{F}^{i}$-measurable and strictly positive: $e^{i}(\omega) \gg 0$ for all $\omega \in \Omega$.

The assumption of strict positivity of initial endowments is often replaced by $\sum_{i=1}^{n} e_{i}(\omega) \gg 0$ for all $\omega \in \Omega$, together with "irreducibility" (i.e., the endowment of every coalition is desired).

Let $L_{X^{i}}$ denote the set of all $\mathcal{F}^{i}$-measurable selections from the random consumption set of agent $i$, that is:

$$
L_{X^{i}}=\left\{x^{i}: \Omega \rightarrow \mathbb{R}_{+}^{l} \text {, such that } x^{i} \text { is } \mathcal{F}^{i} \text {-measurable }\right\} .
$$

And let $L_{X}=\prod_{i=1}^{n} L_{X^{i}}$. Any element $x$ in $L_{X}$ is called an allocation. With "free disposal', an allocation $x \in L_{X}$ is said to be feasible if:

$$
\sum_{i=1}^{n} x^{i} \leq \sum_{i=1}^{n} e^{i} \text { for }(\mu-) \text { almost every } \omega \in \Omega \text {. }
$$

The economic agents seek to maximize their ex-ante expected utility, given by:

$$
U^{i}(x)=\int_{\Omega} u^{i}\left(\omega, x^{i}(\omega)\right) \cdot d \mu .
$$

A coalition $S \subset N$ privately blocks an allocation $x \in L_{X}$ if there exists $\left(y^{i}\right)_{i \in S} \in$ $\prod_{i \in S} L_{X^{i}}$ such that:

$$
\sum_{i \in S} y^{i} \leq \sum_{i \in S} e^{i} \text { and } U^{i}\left(y^{i}\right)>U^{i}\left(x^{i}\right) \text { for every } i \in S .
$$

The private core of a differential information economy $\mathcal{E}$ is the set of all feasible allocations which are not privately blocked by any coalition. Observe that although coalitions of agents are formed, information is not shared between them. 
On the contrary, the redistribution of the initial endowments is based only on each agent's private information.

In the context of a differential information economy, a price system is a $\mathcal{B}$ measurable, non-zero function $\pi: \Omega \rightarrow \mathbb{R}_{+}^{\ell}$.

For a price system $\pi$, the budget set of agent $i$ is given by:

$$
B^{i}\left(\pi, e^{i}\right)=\left\{x^{i} \in L_{X^{i}}, \text { such that } \int_{\Omega} \pi(\omega) x^{i}(\omega) d \mu \leq \int_{\Omega} \pi(\omega) e^{i}(\omega) d \mu\right\} .
$$

A pair $(\pi, x)$ is a Radner equilibrium if $\pi$ is a price system and $x=\left(x^{1}, \ldots, x^{n}\right) \in$ $L_{X}$ is a feasible allocation such that, for every $i, x^{i}$ maximizes $U^{i}$ on $B^{i}\left(\pi, e^{i}\right)$. Notice that traders must balance the budget in the first period. Radner equilibrium is an ex-ante concept.

\section{A Topology on Private Information}

The previous studies on the continuity of economic behavior with respect to information (Allen (1983) and Einy et al (2004)) used the topology introduced by Boylan (1971). This topology is generated by a pseudometric $d$ that assigns a finite distance to any pair of $\sigma$-algebras, $x$ and $y$, contained in $\mathcal{B}$.

$$
d(x, y)=\sup _{A \in x} \inf _{B \in y} \mu(A \Delta B)+\sup _{B \in y} \inf _{A \in x} \mu(A \Delta B) .^{3}
$$

Recall that in our model the information of each agent is a $\sigma$-algebra, $x$, generated by a finite partition of $\Omega$ such that the agent can tell in which of the sets of the partition lies the actual state of nature.

Let $\mathcal{X}=\{x \subset \mathcal{B} ; x$ is the $\sigma$-algebra generated by a finite partition of $\Omega\}$.

\footnotetext{
${ }^{3} A \Delta B$ is the symmetric difference between sets $A$ and $B: A \Delta B \equiv A \backslash B \cup B \backslash A$.
} 
In this paper, we construct a finer topology than Boylan's. The central concept that is used is the "common information". Given two $\sigma$-algebras, $x, y \in \mathcal{X}$, the $\sigma$-algebra that represents the "common information" is defined as:

$$
x \wedge y=\{A \in x: \exists B \in y \text { s.t. } \mu(A \Delta B)=0\} .
$$

Observe that, in general, $x \wedge y \neq y \wedge x$. But also that $d(x \wedge y, y \wedge x)=0$.

To generate the topology, we use a function, $d^{*}: \mathcal{X} \times \mathcal{X} \rightarrow \mathbb{R}^{+}$, defined as the sum of the Boylan distances from each information set to the common information.

Definition $1 \forall x, y \in \mathcal{X}, d^{*}(x, y)=d(x, x \wedge y)+d(x \wedge y, y)$, where $d(x, y)$ is the Boylan distance between the information fields.

This function is not a distance, but a related concept that we designate as a "detachment", since it satisfies the three following properties for all $x, y \in \mathcal{X}$ :

1. Positivity: $d^{*}(x, y) \geq 0$ and $d^{*}(x, x)=0$;

2. Symmetry: $d^{*}(x, y)=d^{*}(y, x){ }^{4}$

3. Discrimination: $d^{*}(x, y)=0$ implies that for every set in $x$ there is a set in $y$ that differs from it by at most a subset of $\Omega$ with $\mu$-measure zero;

The "detachment" falls short of being a pseudometric because it violates the triangle inequality: $d^{*}(x, y) \leq d^{*}(x, z)+d^{*}(z, y) \quad \forall x, y, z \in \mathcal{X}$.

Observe that $d^{*}$ defines an equivalence relation on $\mathcal{X}$. We say that two $\sigma$-algebras $x, x^{\prime} \in \mathcal{X}$ are equivalent if and only if they have a null detachment $\left(x \sim x^{\prime} \Leftrightarrow\right.$ $\left.d^{*}\left(x, x^{\prime}\right)=0\right)$. Let $\mathcal{F}=\mathcal{X} / \sim$ denote the set of equivalence classes of $\mathcal{X}$, that

\footnotetext{
${ }^{4}$ To see this, use $d(x \wedge y, y \wedge x)=0$.
} 
is, $\mathcal{F}=\{[x]: x \in \mathcal{X}\}$, where $[x]=\left\{y \in \mathcal{X}: d^{*}(x, y)=0\right\}$. According to the next proposition, Boylan's pseudometric, $d$, defines exactly the same equivalence classes.

Proposition $3.1 \forall x, y \in \mathcal{X}, d^{*}(x, y)=0 \Leftrightarrow d(x, y)=0$.

Proof. Since $d$ is nonnegative and satisfies the triangle inequality, we have: $0 \leq d(x, y) \leq d^{*}(x, y)$.

On the other hand, $d(x, y)=0$ implies that for every set $A \in x$ there exists a set $B \in y$ such that $\mu(x \Delta y)=0$. But this also means that $x \wedge y=x$. So, $d^{*}(x, y)=d(x, x)+d(x, y)=0+0$.

We use "open balls", $B^{*}(x, \epsilon)=\left\{y \in \mathcal{X}: d^{*}(x, y)<\epsilon\right\}$, to generate the topology. In the case of a metric, the triangle inequality ensures that the open balls generate a topology, that is, that the open sets are arbitrary unions of open balls. In this case, we must actually prove that the "open balls" produced by $d^{*}$ also generate a topology. ${ }^{5}$ This is done in three steps, each of them illustrative of the characteristics of the topology. Proposition 3.2 shows that in a small "open ball", all information partitions have more information than the center. And according to proposition 3.4, in a small "open ball" all partitions have the same common information with a third partition. These two results allow us to prove that a kind of local triangle inequality holds, implying that the "open balls" generated by $d^{*}$ are open sets.

We proceed to show that all information fields that are very close to the finite $\sigma$-algebra $x$ have more information than $x$ :

\footnotetext{
${ }^{5}$ We opted to generalize the concept of "ball" from distances to "detachment", but, alternatively, a different designation can be used, like "open zone" with some "reach" around a center.
} 
Proposition $3.2 \forall x \in \mathcal{X}, \exists \delta(x)>0$ such that

$d^{*}(x, y)<\delta(x) \Rightarrow x \wedge y=x$.

Proof. Consider an $x \in \mathcal{X}$. Since $x$ is a finite $\sigma$-algebra, there exists a finite number of $\sigma$-algebras that are contained in $x$. Thus:

$$
\min _{\substack{z \subset x \\[z] \neq[x]}} d(x, z)=\delta(x)>0 .
$$

By definition, $d^{*}(x, y)<\delta(x) \Rightarrow d(x, x \wedge y)<\delta(x)$.

Since $x \wedge y \subseteq x \Rightarrow[x \wedge y]=[x]$. The way in which $x \wedge y$ is defined implies that $x \wedge y=x$.

The distance of Boylan and our "detachment" are locally equivalent in the following sense.

Proposition $3.3 \forall x \in \mathcal{X}, \exists \delta(x)>0$ such that

$d^{*}(x, y)<\delta(x) \Rightarrow d^{*}(x, y)=d(x, y)$.

Proof. By definition, $d^{*}(x, y)=d(x, x \wedge y)+d(x \wedge y, y)$.

In the small neighborhood as defined by proposition $3.2, x \wedge y=x$.

So, we have: $d^{*}(x, y)=0+d(x, y)$.

Observe that $\delta$ defines a $d^{*}$-ball where this equality holds, but not a $d$-ball. It is not true that $\exists \delta(x)>0$ such that $d(x, y)<\delta(x) \Rightarrow d^{*}(x, y)=d(x, y)$.

Note also that proposition 3.1 is a particular case of proposition 3.3.

From the following proposition we find that given two information fields, if one of them varies slightly, the common information remains the same. 
Proposition $3.4 \forall x, y \in \mathcal{X}, \exists \delta(x, y)>0$ such that $d^{*}(y, z)<\delta(x, y) \Rightarrow x \wedge y=x \wedge z$.

Proof. Consider two arbitrary finite $\sigma$-algebras $x, y \in \mathcal{X}$. Consider first the case $[x]=[y]$. Of course that $x \wedge y=x$. By proposition 3.2, there exists a $\delta(y)$ such that $d^{*}(y, z)<\delta(y)$ implies that $y \wedge z=y$. It follows from the definition of common information that this operation is associative. So, we have $x \wedge y=x \wedge(y \wedge z)=(x \wedge y) \wedge z=x \wedge z$.

With $[x] \neq[y]$, and because we are dealing with finite $\sigma$-algebras, there is only a finite number of subsets of $\Omega, A_{x} \in x$ and $A_{y} \in y$. Thus:

$$
\min _{\mu\left(A_{x} \Delta A_{y}\right)>0} \mu\left(A_{x} \Delta A_{y}\right)=\epsilon .
$$

Given $x$ and $y$, consider $\delta(y)$ as in proposition 3.2 and let $\delta(x, y)=\min \{\epsilon, \delta(y)\}$. From proposition $3.2, d^{*}(y, z)<\delta(x, y) \Rightarrow y=y \wedge z$.

This implies that $\Rightarrow x \wedge y=x \wedge y \wedge z$.

If we assume that $x \wedge z \neq x \wedge y \wedge z$, then, by the way we defined the "common information", we are sure that $[x \wedge z] \neq[x \wedge y \wedge z]$. So there exist $A_{z} \in z$ and $A_{x} \in x$ with $\mu\left(A_{z} \Delta A_{x}\right)=0$ such that there isn't any $A_{y} \in y$ with $\mu\left(A_{z} \Delta A_{y}\right)=0$ or $\mu\left(A_{x} \Delta A_{y}\right)=0$. So, $\min _{A_{y} \in y} \mu\left(A_{z} \Delta A_{y}\right) \geq \epsilon \geq \delta(x, y)$.

This implies that $d^{*}(y, z) \geq d(y, z) \geq \min _{A_{y} \in y} \mu\left(A_{z} \Delta A_{y}\right) \geq \delta(x, y)$.

We have a contradiction, so $x \wedge z=x \wedge y \wedge z=x \wedge y$.

We can read propositions 3.2 and 3.4 together. By the first, in a small neighborhood of an information set, information does not decrease. According to the second, the possible increase of information has a bound, as the common information with another information set remains constant.

Theorem 1 is based on a kind of local triangle inequality which implies that all the points of an "open ball" are "interior points". A point $x$ is "interior" to $A \Leftrightarrow$ 
$\exists \epsilon>0$ s.t. $B^{*}(x, \epsilon) \subset A$. As a consequence, the collection of the "open balls" is a base for the topology $\tau^{*}=\{A: \mathrm{A}$ is a union of open balls $\}$.

Theorem $1 \forall x \in \mathcal{X}$ and $\epsilon>0: B^{*}(x, \epsilon)=\left\{y \in \mathcal{X}: d^{*}(x, y)<\epsilon\right\}$ is open (all points are interior). The collection of open balls with rational radius is a base for $\tau^{*} .\left(\mathcal{X}, \tau^{*}\right)$ is a topological space.

Proof. Given an arbitrary ball $B^{*}(x, \epsilon)$, we want to show that all points of this ball are interior points. Equivalently, that given $y \in B^{*}(x, \epsilon)$, there exists $\delta^{\prime}(x, y)>0$ such that $B^{*}\left(y, \delta^{\prime}(x, y)\right) \subset B^{*}(x, \epsilon)$.

Consider some $\delta(x, y)$ small enough for proposition 3.4 to hold, and an arbitrary $z \in B^{*}(y, \delta(x, y))$.

$$
\begin{aligned}
d^{*}(x, z) & =d(x, x \wedge z)+d(x \wedge z, z)=(\text { by proposition 3.4) } \\
& =d(x, x \wedge y)+d(x \wedge y, z) \leq \\
& \leq d(x, x \wedge y)+d(x \wedge y, y)+d(y, z) \leq \\
& \leq d^{*}(x, y)+d(y, z) \leq \\
& \leq d^{*}(x, y)+d^{*}(y, z) .
\end{aligned}
$$

Let $\delta^{\prime}(x, y)=\min \left\{\delta(x, y), \epsilon-d^{*}(x, y)\right\}$. For any $z \in B^{*}\left[y, \delta^{\prime}(x, y)\right]$, we have:

$$
d^{*}(x, z) \leq d^{*}(x, y)+d^{*}(y, z) \leq d^{*}(x, y)+\epsilon-d^{*}(x, y)=\epsilon .
$$

Thus, the arbitrary $y$ is an interior point. All points in $B^{*}(x, \epsilon)$ are interior points.

The sets whose points are all interior are open sets (members of the topology), since they can be obtained by arbitrary unions of the members of the base. To see this, consider an arbitrary set A, whose points are all interior.

$$
\begin{aligned}
& A=\operatorname{int}(A) \Rightarrow \forall x \in A, \exists B^{*}\left(x, \epsilon_{x}\right) \subset A \Rightarrow \\
& \Rightarrow \cup_{x \in A} B^{*}\left(x, \epsilon_{x}\right) \subset A \subset \cup_{x \in A} B^{*}\left(x, \epsilon_{x}\right) \Rightarrow A \text { is a union of open balls. }
\end{aligned}
$$


Of course that all the points inside an open set are interior points. A point in a set that is an union of open sets is interior to at least one of the open sets, therefore it is also interior to the union.

For the topology to be well defined, a finite intersection of open sets $A$ must be open. It is enough to prove that the intersection of two open sets is open. Consider an arbitrary point $a \in A=A_{1} \cap A_{2}$. The point is interior to both open sets, so each of them contains a ball centered in $a$. Designate these balls by $B^{*}\left(a, r_{1}\right) \subset A_{1}$ and $B^{*}\left(a, r_{2}\right) \subset A_{2}$. Pick the smallest radius, w.l.o.g., $r_{1}$. Of course that $B^{*}\left(a, r_{1}\right) \subset A_{1}$ and $B^{*}\left(a, r_{1}\right) \subset A_{2}$. This open ball, $B^{*}\left(a, r_{1}\right)$, is contained in the intersection.

The Boylan topology, defined by $d$, is a Hausdorff topology on the space of equivalence classes of information $\sigma$-algebras. This topology is finer, so it inherits this property. ${ }^{6}$ Observe that the topology is first countable, as every point has a countable neighborhood base. ${ }^{7}$ Thus, to prove upper semicontinuity of the equilibrium (or private core) correspondence, it suffices to show that given a convergent sequence of economies, the limit of a sequence of equilibrium (private core) allocations of the sequence of economies is an equilibrium (private core) allocation of the limit economy (see theorem 16.20 of Aliprantis and Border (1999)).

\footnotetext{
${ }^{6}$ Given any two distinct points, there are Boylan neighborhoods of each point with null intersections (the Boylan and Cotter topologies are separated because every topology generated by a metric is separated). And for every Boylan neighborhood, there is a neighborhood in this topology that is contained in it, because: $\forall x \in \mathcal{X}, \epsilon \in R^{+}: B^{*}(x, \epsilon) \subset B(x, \epsilon)$. This implies that this topology is also separated.

${ }^{7}$ For every neighborhood of any $x$, there is an open ball with rational radius centered in $x$ that is contained in the neighborhood.
} 


\section{Upper Semicontinuity of the Private Core}

In this section we establish upper semicontinuity of the private core correspondence. First we make precise the notion of convergence of economies. Then we show that given a convergent sequence of economies, the limit of a sequence of private core allocations of the sequence of economies belongs to the private core of the limit economy.

Definition 2 Let $\left\{\mathcal{E}_{k}\right\}_{k=1}^{\infty} \equiv\left\{\left(e_{k}^{i}, u_{k}^{i}, F_{k}^{i}\right)_{i=1}^{n}\right\}_{k=1}^{\infty}$ be a sequence of economies with differential information that converges to $\mathcal{E}_{0} \equiv\left(e_{0}^{i}, u_{0}^{i}, F_{0}^{i}\right)_{i=1}^{n}$. Precisely, convergence means that, for every agent $i \in N$ :

i) $e_{k}^{i}$ converges to $e_{0}^{i}$ in the $L_{1}^{\ell}$-norm;

ii) $u_{k}^{i}$ converges uniformly to $u_{0}^{i}$ on every compact subset of $\Omega \times \mathbb{R}_{+}^{\ell}$;

iii) $F_{k}^{i}$ converges to $F_{0}^{i}$ in $\left(\mathcal{X}, \tau^{*}\right)$.

Upper semicontinuity of the Radner equilibrium set and of the private core can be established with the use of two technical lemmas from Einy et al (2004). The first states that the limit of informationally feasible functions is also informationally feasible.

Lemma 4.1 Let $\left\{F_{k}\right\}_{k=1}^{\infty}$ be a sequence of $\sigma$-subfields of $\mathcal{F}$ that converges to $\mathcal{F}_{0}$ in Boylan metric, and let $\left\{x_{k}\right\}_{k=1}^{\infty}$ be a sequence of allocations in $L_{1}^{\ell}(\Omega, \mathcal{F}, \mu)$ that converges to $x_{0}$ in $L_{1}^{\ell}$-norm.

If for every $k, x_{k}$ is $F_{k}$-measurable, then $x_{0}$ is $F_{0}$-measurable. 
The second lemma states that the limit of a sequence of utilities equals the limit utility of the limit allocation. With the consideration of finite partitions of information, the similar lemma from Einy et al (2004) that assumes continuous utility functions in $\Omega \times \mathbb{R}_{+}^{\ell}$ can easily be extended. It is enough to assume that, given $x$, the utility is bounded and continuous in the interior of sets of the agent's partition of information.

Lemma 4.2 Let $\left\{u_{k}\right\}_{k=1}^{\infty}$ be a sequence of random utility functions on $\Omega \times \mathbb{R}_{+}^{\ell}$. For all $k$ and all $\omega \in \Omega, u_{k}(\omega, \cdot)$ is continuous, concave, non-negative and nondecreasing. For every $x, u_{k}(\cdot, x): \Omega \rightarrow \mathbb{R}_{+}$is measurable in $\mathcal{F}$, bounded, and continuous in the interior of the sets of a finite partition of $\Omega$. Assume that $\left\{u_{k}\right\}_{k=1}^{\infty}$ converges to a function $u_{0}$ uniformly on every compact subset of $\Omega \times \mathbb{R}_{+}^{\ell}$. In these conditions, if $\left\{x_{k}\right\}_{k=1}^{\infty}$ is a sequence in $L_{1}^{\ell}$ that converges to $x_{0}$ in the $L_{1}^{\ell}$-norm, then:

$$
\lim _{k \rightarrow \infty} \int_{\Omega} u_{k}\left(\omega, x_{k}(\omega)\right) \cdot d \mu(\omega)=\int_{\Omega} u_{0}\left(\omega, x_{0}(\omega)\right) \cdot d \mu(\omega)
$$

The following theorem, from Einy, Haimanko, Moreno and Shitovitz (2004), establishes upper semicontinuity of the Radner equilibrium correspondence.

Theorem 2 Let $\left\{\mathcal{E}_{k}\right\}_{k=1}^{\infty} \equiv\left\{\left(e_{k}^{i}, u_{k}^{i}, F_{k}^{i}\right)_{i=1}^{n}\right\}_{k=1}^{\infty}$ be a sequence of economies with differential information that converges to $\mathcal{E}_{0} \equiv\left(e_{0}^{i}, u_{0}^{i}, F_{0}^{i}\right)_{i=1}^{n}$.

If $\left\{\left(x_{k}, \pi_{k}\right)\right\}_{k=1}^{\infty}$ is a sequence such that $\left(x_{k}, \pi_{k}\right) \in R E\left(\mathcal{E}_{k}\right)$ for every $k$, and for every $i \in N$ :

i) $x_{k}^{i}$ converges to $x_{0}^{i}$ in the $L_{p}^{\ell}$-norm;

ii) $\pi_{k}^{i}$ converges to $\pi_{0}^{i}$ in the $L_{q}^{\ell}$-norm; 
then $\left(x_{0}, \pi_{0}\right) \in R E\left(\mathcal{E}_{0}\right)$.

Theorem 3 combines a result of Einy et al (2004) with the topology that we introduce (lemma 3.1) to establish upper semicontinuity of the private core correspondence.

Theorem 3 Let $\left\{\mathcal{E}_{k}\right\}_{k=1}^{\infty} \equiv\left\{\left(e_{k}^{i}, u_{k}^{i}, F_{k}^{i}\right)_{i=1}^{n}\right\}_{k=1}^{\infty}$ be a sequence of economies with differential information that converges to $\mathcal{E}_{0} \equiv\left(e_{0}^{i}, u_{0}^{i}, F_{0}^{i}\right)_{i=1}^{n}$.

If a convergent sequence of allocations in $L_{1}^{\ell},\left\{x_{k}\right\}_{k=1}^{\infty}$, with $\lim _{k \rightarrow \infty} x_{k}=x_{0}$, is such that, for every $k, x_{k}=\left(x_{k}^{1}, x_{k}^{2}, \ldots, x_{k}^{n}\right)$ is a private core allocation in $\mathcal{E}_{k}$, then the limit of the sequence, $x_{0}=\left(x_{0}^{1}, x_{0}^{2}, \ldots, x_{0}^{n}\right)$, is a private core allocation in $\mathcal{E}_{0}$.

The proof is straightforward from lemma 3.1, lemma 4.2 and theorem 2 of Einy, Haimanko, Moreno and Shitovitz (2004). 


\section{Concluding Remarks}

In the introduction, we mentioned an example presented by Einy, Haimanko, Moreno and Shitovitz (2004) that excluded upper semicontinuity of the private core. With our topology, the sequence of economies considered in that example does not converge. We reproduce the example below.

Consider a sequence of economies, $\mathcal{E}_{\epsilon}$, with two agents and one commodity, where only one of the private information fields varies. The space of possible states of nature is $\Omega=[0,1] \cup[2,3]$. The agents have equal initial endowments, independent of the state of nature: $e(\omega)=\frac{1}{2}$. The private information of the agents are the finite fields generated by the partitions: $\left\{\begin{array}{l}F_{\epsilon}^{1}=[0,1] \cup[2,2+\epsilon],(2+\epsilon, 3] \\ F_{\epsilon}^{2}=[0,1],[2,3]\end{array}\right.$. Agent 1 only values consumption only in $[0,1]$, while agent 2 values consumption only in $[2,3]$. Their preferences are: $u^{1}(\omega, x)=\left\{\begin{array}{l}x, \text { if } \omega \in[0,1] \\ 0, \text { if } \omega \in[2,3]\end{array}\right.$, and $u^{2}(\omega, x)=\left\{\begin{array}{ll}0 & , \text { if } \omega \in[0,1] \\ x & \text {, if } \omega \in[2,3]\end{array}\right.$.

The economies differ only in the parameter $\epsilon$, which converges to zero. Private allocations are of the form:

$x_{\epsilon}=\left(a_{\epsilon}^{1} \cdot \chi_{[0,1] \cup[2,2+\epsilon]}+a_{\epsilon}^{2} \cdot \chi_{(2+\epsilon, 3]}, b_{\epsilon}^{1} \cdot \chi_{[0,1]}+b_{\epsilon}^{2} \cdot \chi_{[2,3]}\right)$.

Feasibility in $[0,1],[2,2+\epsilon]$, and in $(2+\epsilon, 3]$ implies that:

$$
\left\{\begin{array}{l}
a_{\epsilon}^{1}+b_{\epsilon}^{1} \leq 1 \\
a_{\epsilon}^{1}+b_{\epsilon}^{2} \leq 1 \\
a_{\epsilon}^{2}+b_{\epsilon}^{2} \leq 1
\end{array}\right.
$$

Since $x_{\epsilon}$ is a core allocation, $a_{\epsilon}^{1} \geq \frac{1}{2}$, or else $U^{1}\left(e^{1}\right)>U^{1}\left(x_{\epsilon}^{1}\right)$. For the same reason, $b_{\epsilon}^{2} \geq \frac{1}{2}$. So, $a_{\epsilon}^{1}+b_{\epsilon}^{2} \leq 1$ implies that $a_{\epsilon}^{1}=b_{\epsilon}^{2}=\frac{1}{2}$, that $b_{\epsilon}^{1} \leq \frac{1}{2}$, and that $a_{\epsilon}^{2} \leq \frac{1}{2}$. 
Therefore, the initial endowments form a (constant) sequence of private core allocations, converging, of course, to $e=\frac{1}{2}$. In the limit economy, where $F^{1}=F^{2}=$ $\{[0,1],[2,3]\}, x_{\epsilon}=\left(\chi_{[0,1]}, \chi_{[2,3]}\right)$ is the only private core allocation, corresponding to a situation in which agent 1 consumes everything in $[0,1]$ and agent 2 consumes everything in $[2,3]$. Upper semicontinuity of the private core correspondence fails.

Observe that in the sequence of economies $\mathcal{E}_{\epsilon}$, even for a very small $\epsilon$, the common information of the agents is null. So, agent 1 cannot trade consumption in $[2,3]$ (which has no value) for consumption in $[0,1]$ (which agent 2 doesn't value). Their information fields are incompatible, in the sense that they do not allow trades that are contingent on the states of nature. In the limit economy the agents have the same information, so they are able to make contingent trades. According to Boylan's topology on information, the fields $[0,1] \cup[2,2+\epsilon],(2+\epsilon, 3]$ and $[0,1],[2,3]$ are neighbor. Nevertheless, the first information is useless for agent 1 , while the second, being compatible with the information of agent 2 , is valuable.

With our topology, there is no failure of continuity, because the sequence of information fields $F_{\epsilon}^{1}=[0,1] \cup[2,2+\epsilon],(2+\epsilon, 3]$ does not converge. A sequence that would actually converge to $F_{0}^{1}=[0,1],[2,3]$ in our topology is, for example, $F_{\epsilon}^{1^{\prime}}=[0,1],[2,2+\epsilon],(2+\epsilon, 3]$. But, in this case, contingent trades would be allowed in the sequence of economies, and not just in the limit economy.

In our topology, two information fields that are neighbor may differ only in events that are very unlikely. Notice that $F_{0}^{1}$ and $F_{\epsilon}^{1^{\prime}}$ differ because while $F_{0}^{1}$ observes $[2,3], F_{\epsilon}^{1^{\prime}}$ can distinguish the unlikely event $[2,2+\epsilon]$ from $(2+\epsilon, 3]$. Trades contingent on realization of $[2,3]$ are allowed. Only trades that are contingent on a very unlikely event, $[2,2+\epsilon]$, are excluded.

Information fields that are very close in the topology of Boylan may also differ in an additional way, by distinguishing different but very correlated events. In fact, $F_{0}^{1}$ and $F_{\epsilon}^{1}$ differ because they allow the observation of very correlated events: 
$[0,1] \cup[2,2+\epsilon]$ is similar to $[0,1]$; and $(2+\epsilon, 3]$ is similar to $[2,3]$. Nevertheless, the common information is null and so contingent agreements are not allowed.

The differences of the first kind only imply that agreements cannot be contingent on the very unlikely events that ere not commonly observes, and therefore, have a small impact on economic outcomes. Differences of the second kind may prevent valuable agreements, contingent on events that are not commonly observed but nevertheless probable, and thus may imply very different economic outcomes. We reiterate that this second type of differences between information fields that are neighbor is allowed by the topology of Boylan but not by the topology that we introduce.

\section{References}

Aliprantis, C. and Border, K. (1999), "Infinite Dimensional Analysis", 2nd ed., New York: Springer, 1999.

Allen, B. (1983), "Neighboring Information and Distribution of Agent Characteristics under Uncertainty", Journal of Mathematical Economics, 12, pp. 63-101.

Boylan, E. (1971), "Equiconvergence of Martingales", Annals of Mathematical Statistics, 42, pp. 552-559.

Cotter, K.D. (1986), "Similarity of Information and Behavior with Pointwise Convergence Topology", Journal of Mathematical Economics, 15, pp. 25-38.

Cotter, K.D. (1987), "Convergence of Information, Random Variables and Noise", Journal of Mathematical Economics, 16, pp. 39-51.

Debreu, G. (1959), "Theory of Value," Yale University press, New Haven, 1959.

Einy, E., Haimanko, O., Moreno, D. and Shitovitz, B. (2004), "On the Continuity of Equilibrium and Core Correspondences in Economies with Differential Information", Working Papers 03-27, Economic Series 07, Universidad Carlos III de Madrid (January 2004). 
Einy, E., Moreno, D. and Shitovitz, B. (2001), "Competitive and Core Allocations in Large Economies with Differentiated Information", Economic Theory, 18, 321-332.

Einy, E. and Shitovitz, B. (2002), "Private Value in Large Economies with Differential Information", Games and Economic Behavior, 34, 287-311.

Glycopantis, D., Muir, A. and Yannelis, N.C. (2001), "An Extensive Form Interpretation of the Private Core", Economic Theory, 18, pp. 293-319.

Hervés-Beloso, C., Moreno, E. and Yannelis, N.C. (2003), "An Equivalence Theorem for a Differential Information Economy", to appear in Journal of Mathematical Economics (2004).

Hildenbrand, W. and Mertens, J.F. (1972), "Upper Hemi-Continuity of the EquilibriumSet Correspondence for Pure Exchange Economies", Econometrica, Vol. 40, 1, pp. 99-108.

Kannai, Y. (1970), "Continuity Properties of the Core of a Market", Econometrica, 38, 6, pp. $791-815$.

Radner, R. (1968), "Competitive Equilibrium under Uncertainty", Econometrica, 36, pp. $31-58$.

Radner, R. (1982), "Equilibrium under Uncertainty", in K.J. Arrow, M.D. Intriligator (eds.) Handbook of Mathematical Economics, vol. II, Amsterdam: North-Holland.

Yannelis, N.C. (1991), "The Core of an Economy with Differential Information", Economic Theory, 1, 183-198. 